\section{Influence of cycloplegia with topical cyclopentolate on higher-order aberrations in myopic children}

T Hiraoka ${ }^{1}$, K Miyata ${ }^{2}$, Y Nakamura ${ }^{2}$, M Ogata $^{2}$, F Okamoto ${ }^{1}$ and T Oshika ${ }^{1}$

\begin{abstract}
Purpose To investigate the influence of cycloplegia with topical cyclopentolate on wavefront aberrations in myopic children. Design This is a prospective, comparative study.
\end{abstract}

Methods Twenty-eight myopic children with a mean age of $7.25 \pm 2.55$ were enrolled in this study. We evaluated refraction and wavefront aberrations before and after cycloplegia with $1 \%$ cyclopentolate hydrochloride. Ocular and corneal aberrations were simultaneously measured and compared with each other. Individual Zernike components were also analyzed up to the sixth order. All these parameters were compared before and after cycloplegia.

Results Ocular higher-order aberrations (HOAs) significantly increased after cycloplegia ( $P=0.012$ for spherical-like and $P=0.015$ for total HOAs). Corneal HOAs did not change after cycloplegia. When corneal and ocular HOAs were compared, the ocular HOAs were significantly smaller than the corneal HOAs in spherical-like aberrations $(P<0.001)$ and total HOAs $(P=0.006)$. As for individual Zernike components, ocular aberration generally showed smaller or equivalent values in comparison with corneal aberration. In addition, each Zernike component showed a large standard deviation.

Conclusions Internal optics compensates for corneal HOAs in myopic children, and paralysis of tonic accommodation with cyclopentolate considerably affects ocular HOAs. However, inter-individual variation in each Zernike component is quite large in myopic children.
Eye (2014) 28, 581-586; doi:10.1038/eye.2014.34; published online 28 February 2014

\section{Introduction}

Wavefront analysis is a sophisticated method to accurately evaluate refractive errors. It enables us to obtain information regarding not only lower-order aberrations (defocus and astigmatism) but also higher-order aberrations (HOAs) that cannot be corrected by spherocylindrical lenses. HOAs are important factors to determine retinal image quality, and recent advances in wavefront and laser technologies have allowed for the surgical correction of HOAs and lower-order aberrations. Among the individual HOA components, coma and spherical aberration are common and proven to considerably affect the quality of vision, ${ }^{1,2}$ but various other components of HOAs have been shown to degrade retinal image quality, especially when the pupil dilates. ${ }^{3}$ On the basis of these findings, increasing attention has been directed to the evaluation of ocular optical quality using wavefront aberrometry in the context of various diseases and surgeries, and the clinical importance has been well recognized. ${ }^{1-3}$

Thus far, numerous fundamental studies on ocular wavefront aberrations have been carried out, and some of them have elucidated that HOAs increase with age. ${ }^{4-6}$ However, most studies have been conducted in adults and the information on HOAs in a pediatric population is still very limited. ${ }^{6-9}$

The influence of accommodation on HOAs has also been investigated,, $10-14$ and previous studies have shown that HOAs change with
${ }^{1}$ Department of Ophthalmology, Faculty of Medicine, University of Tsukuba, Ibaraki, Japan

${ }^{2}$ Miyata Eye Hospital, Miyazaki, Japan

Correspondence: T Hiraoka, Department of Ophthalmology, Faculty of Medicine, University of Tsukuba, 1-1-1 Tennoudai, Tsukuba, Ibaraki 305-8575, Japan

Tel: +81 29853 3148;

Fax: +8129853 3148

E-mail: thiraoka@

md.tsukuba.ac.jp

Received: 11 August 2013 Accepted in revised form: 15 January 2014 Published online: 28 February 2014 
increasing levels of accommodation. ${ }^{10,12}$ However, very few attempts have been made to clarify the influence of cycloplegia on HOAs. Carkeet et $a l^{7}$ investigated the differences in ocular HOAs as determined on the basis of cycloplegic and non-cycloplegic measurements in young adults aged 18-27 years, and reported that total HOAs in the cycloplegic condition were slightly higher than in the non-cycloplegic condition. With respect to a pediatric population, Kirwan et $a l^{15}$ showed that cycloplegia with cyclopentolate had little effect on HOAs in children aged from 4 to 14 years. However, there is no other information available regarding the cycloplegic effect of cyclopentolate on HOAs in children.

Cycloplegic refraction is a useful and essential clinical technique for the examination of infants, children, and patients with suspected accommodative anomalies. ${ }^{16}$ The most commonly used agent is 0.5 and $1 \%$ cyclopentolate, which is a muscarinic antagonist that paralyzes the actions of the ciliary muscle. Although the topical administration of cyclopentolate is quite frequently used in clinical practice to achieve an accurate refraction, the exact influence on HOAs has not been sufficiently investigated in children. In addition, it may be helpful to know the primary state of HOAs after removal of accommodative effects for a better understanding of ocular development in children. Recently, we investigated the influence of cycloplegia with topical atropine on ocular aberrations in hyperopic children, and found a significant increase in ocular HOAs after cycloplegia. ${ }^{17}$ However, in that study, we did not include myopic children, and the effect of cyclopentolate was not examined. Therefore, we conducted the current prospective study to elucidate the impact of cycloplegia with topical cyclopentolate on ocular aberrations in a pediatric population with myopia.

\section{Materials and methods}

Subjects were recruited between July 2009 and January 2010 at the Miyata Eye Hospital. They were selected from consecutive cases among the clinic population who matched our inclusion criteria. The inclusion criteria were as follows: age between 3 and 12 years, eyes with myopic refractive errors, best-corrected visual acuity (BCVA) of 20/25 or better, and no ocular or systemic pathology except refractive errors and/or ocular deviation. Subjects were excluded from the study if they did not cooperate for aberrometry or if their parents refused to allow the instillation of cyclopentolate.

Subjects with pupil diameter of less than $6 \mathrm{~mm}$ in a dark room were also excluded. A total of 28 subjects (17 boys and 11 girls) were enrolled in this study. Only the right eye data for each subject were included in the analyses. At baseline, the ages of the study participants ranged from 4 to 12 years $(7.25 \pm 2.55$ years, mean \pm SD). The manifest refraction ranged between -0.75 and -8.00 diopters (D) $(-3.07 \pm 2.23 \mathrm{D})$, with refractive cylinder between 0.00 and $4.50 \mathrm{D}(1.80 \pm 1.40 \mathrm{D})$. All subjects had BCVA of $0.8(20 / 25)$ or better $(-0.01 \pm 0.08$ logarithm of the minimum angle of resolution). The study adhered to the tenets of the Declaration of Helsinki and was approved by the institutional review board of the Miyata Eye Hospital. Every child gave assent to participate in the study, and written informed consent was obtained from the parents or legal guardians after a thorough explanation of the nature and risks of the study, before enrollment.

At the time of enrollment, all subjects underwent comprehensive ophthalmic evaluations including a manifest refraction, uncorrected visual acuity (UCVA) and BCVA measurements, slit-lamp microscopy, and wavefront aberrometry. Three drops of $1 \%$ cyclopentolate hydrochloride (Cyplegin 1\% ophthalmic solution, Santen Pharmaceutical, Osaka, Japan) were instilled at 5-min intervals. The refraction, UCVA, BCVA, and wavefront aberrations were re-evaluated $60 \mathrm{~min}$ after the first instillation.

Ocular wavefront aberrations were measured with a Hartmann-Shack aberrometer (KR-9000PW; Topcon, Tokyo, Japan) in a dark room. Corneal topography was performed simultaneously using a Placido disk attached to the Hartmann-Shack aberrometer. Corneal HOAs were also measured as described previously. ${ }^{18-20}$ Mydriatic agents were not used at the baseline examination to allow for natural accommodation. A built-in target inside the aberrometer was set in the position for infinity. The target was 'fogged' (blurred by additional lenses) to achieve the physiological relaxation of accommodation so that the measurement was captured at a resting tonus. All of the obtained images were revised to pupil-center images. The HOAs were analyzed for a 6-mm pupil up to the sixth order by expanding the set of Zernike polynomials. From the Zernike coefficients, the root mean square (RMS) was calculated for second-, third-, fourth-, and total higherorder aberrations. The second-order aberration corresponds to conventional refractive error (sphere and cylinder), which can be corrected by spherocylindrical lenses, whereas third- and higher-order aberrations cannot. Therefore, third- and greater-order aberrations are identified as HOAs. The RMS of third-order (S3) and fifth-order (S5) Zernike coefficients was used to represent coma-like aberration (S3 + S5), and the RMS of fourthorder (S4) and sixth-order (S6) Zernike coefficients was used to denote spherical-like aberrations (S4+S6). Total HOAs were calculated as the RMS of third-, fourth-, fifth-, and sixth-order Zernike coefficients (S3+S4+S5+S6). The measurements were repeated at least five times for 
each eye. The three best-focused images were selected and averaged. The averaged values were used for subsequent analyses. This examination procedure was repeated after the patient was cyclopleged using $1 \%$ cyclopentolate hydrochloride solution. We also performed an analysis of individual Zernike components up to the sixth order.

The data obtained after cycloplegia were compared with the baseline measurements using the paired $t$-test. Values for which $P$ was $<0.05$ were considered significant. However, significant values for HOAs, such as coma-like, spherical-like, and total HOAs, were set at $P<0.016$, and those for individual Zernike components were set at $P<0.002$ after adjustment for multiple testing using a Bonferroni correction of the Type 1 error. All statistical analyses were performed using the SPSS version $15.0 \mathrm{~J}$ software (SPSS Inc., Chicago, IL, USA).

\section{Results}

Cycloplegia with cyclopentolate significantly changed the spherical equivalent refraction from $-3.07 \pm 2.23$ to $-2.57 \pm 2.15 \mathrm{D}(P<0.001$, paired $t$-test $)$. Ocular HOAs increased significantly from $0.124 \pm 0.041$ to $0.138 \pm 0.037 \mu \mathrm{m}$ for spherical-like aberrations $(P=0.012)$, and from $0.279 \pm 0.105$ to $0.304 \pm 0.096 \mu \mathrm{m}$ for total HOAs $(P=0.015)$. Coma-like aberrations also increased from $0.243 \pm 0.111$ to $0.263 \pm 0.105 \mu \mathrm{m}$, but this change was not statistically significant $(P=0.042)$ (Figure 1a). Corneal HOAs did not change after cycloplegia $(P=0.307$ for coma-like aberrations, $P=0.118$ for spherical-like aberrations, and $P=0.105$ for total HOAs) (Figure $1 \mathrm{~b}$ ). When ocular and corneal HOAs were compared, the ocular HOAs were significantly smaller than the corneal
HOAs in spherical-like aberrations and total HOAs $(P<0.001$ for spherical-like aberrations and $P=0.006$ for total HOAs) (Figure 1c).

Table 1 shows the individual Zernike components from $C_{2}{ }^{-2}$ to $C_{6}{ }^{6}$ before and after cycloplegia. Significant changes were found in the $C_{2}{ }^{0}$ and $C_{4}{ }^{0}$ components. The $C_{2}{ }^{0}$ decreased from $3.881 \pm 0.507$ (mean \pm SE) to $3.268 \pm 0.452 \mu \mathrm{m}(P<0.001)$. The $C_{4}{ }^{0}$ increased from $0.033 \pm 0.015$ to $0.050 \pm 0.015 \mu \mathrm{m}(P=0.001)$. The induced change in each Zernike component was analyzed in relation to the patient's age, baseline refractive error, and induced change in spherical equivalent refraction; however, no significant relationships were observed.

Figure 2 shows the mean value of the corneal and ocular aberrations for individual Zernike components before cycloplegia. In general, ocular aberration was smaller than or equivalent to corneal aberration. There were significant differences in $C_{3}{ }^{1}, C_{4}{ }^{0}, C_{5}{ }^{-1}, C_{5}{ }^{3}, C_{6}{ }^{0}$, and $C_{6}{ }^{2}$ between corneal and ocular aberrations $(P<0.002)$. In particular, horizontal coma $\left(C_{3}{ }^{1}\right)$ and spherical aberration $\left(C_{4}{ }^{0}\right)$ were outstanding.

\section{Discussion}

In this study, we analyzed the optical quality of the eye before and after cycloplegia with cyclopentolate in a pediatric population with myopia. The average of spherical equivalent refraction changed significantly from -3.07 to $-2.57 \mathrm{D}$ after cycloplegia, showing a positive shift of $0.50 \mathrm{D}$. It is known that cycloplegia inhibits accommodation and thereby prevents the overestimation of myopia and the underestimation of hyperopia. ${ }^{21}$ Even when viewing an object at infinity, a certain amount of accommodation (0.25-0.50 D) is present
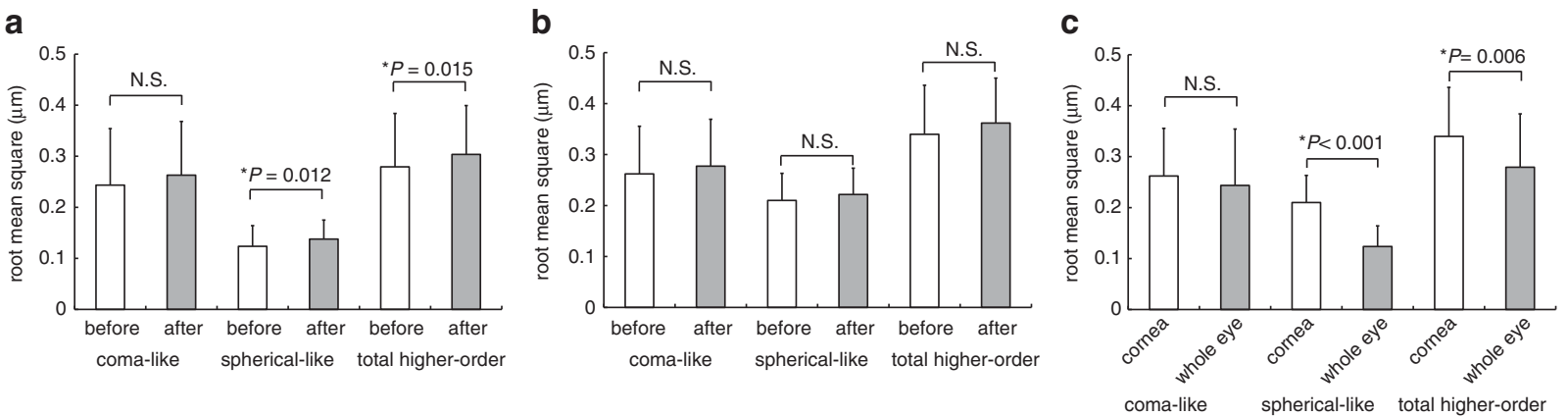

Figure 1 Ocular and corneal HOAs before and after cycloplegia. (a) Ocular HOAs before and after cycloplegia. Ocular HOAs increased significantly from $0.124 \pm 0.041$ to $0.138 \pm 0.037 \mu \mathrm{m}$ for spherical-like aberrations $(P=0.012)$, and from $0.279 \pm 0.105$ to $0.304 \pm 0.096 \mu \mathrm{m}$ for total HOAs $(P=0.015)$. Coma-like aberrations also increased from $0.243 \pm 0.111$ to $0.263 \pm 0.105 \mu \mathrm{m}$, but this change was not statistically significant $(P=0.042)$. Graphs are expressed as the mean \pm SD. (b) Corneal HOAs before and after cycloplegia. Corneal HOAs did not change after cycloplegia $(P=0.307$ for coma-like aberrations, $P=0.118$ for spherical-like aberrations, and $P=0.105$ for total HOAs, paired $t$-test). Graphs are expressed as the mean \pm SD. (c) Comparison of HOAs between the cornea and whole eye before cycloplegia. When corneal and ocular (whole-eye) HOAs were compared, the ocular HOAs were significantly smaller than the corneal HOAs in spherical-like aberrations $(P<0.001$, paired $t$-test $)$ and total HOAs $(P=0.006)$. Graphs are expressed as the mean $\pm \mathrm{SD}$. HOAs, higher-order aberrations. 
Table 1 Change in each Zernike component before and after cycloplegia

\begin{tabular}{|c|c|c|c|}
\hline \multirow[t]{2}{*}{ Zernike component } & \multicolumn{2}{|c|}{ Coefficient $(\mu m)($ mean $\pm S D)$} & \multirow[t]{2}{*}{ P-value } \\
\hline & Before & After & \\
\hline$C_{2}{ }^{-2}$ & $0.032 \pm 0.612$ & $0.015 \pm 0.656$ & 0.625 \\
\hline $\mathrm{C}_{2}{ }^{0}$ & $3.881 \pm 2.685$ & $3.268 \pm 2.394$ & $<0.001^{\mathrm{a}}$ \\
\hline$C_{2}^{2}$ & $-1.269 \pm 1.348$ & $-1.290 \pm 1.337$ & 0.212 \\
\hline$C_{3}^{-3}$ & $0.041 \pm 0.134$ & $0.054 \pm 0.131$ & 0.090 \\
\hline$C_{3}^{-1}$ & $0.018 \pm 0.167$ & $0.038 \pm 0.177$ & 0.056 \\
\hline$C_{3}{ }^{1}$ & $-0.015 \pm 0.100$ & $-0.017 \pm 0.102$ & 0.797 \\
\hline$C_{3}{ }^{3}$ & $-0.056 \pm 0.092$ & $-0.069 \pm 0.100$ & 0.078 \\
\hline$C_{4}^{-4}$ & $0.025 \pm 0.035$ & $0.024 \pm 0.035$ & 0.776 \\
\hline$C_{4}^{-2}$ & $-0.017 \pm 0.030$ & $-0.019 \pm 0.031$ & 0.522 \\
\hline $\mathrm{C}_{4}{ }^{0}$ & $0.033 \pm 0.080$ & $0.050 \pm 0.081$ & $0.001^{\mathrm{a}}$ \\
\hline$C_{4}^{2}$ & $0.013 \pm 0.042$ & $0.003 \pm 0.046$ & 0.037 \\
\hline$C_{4}^{4}$ & $-0.006 \pm 0.037$ & $0.004 \pm 0.048$ & 0.110 \\
\hline$C_{5}{ }^{-5}$ & $-0.016 \pm 0.016$ & $-0.027 \pm 0.024$ & 0.004 \\
\hline$C_{5}^{-3}$ & $0.001 \pm 0.023$ & $0.011 \pm 0.031$ & 0.005 \\
\hline$C_{5}^{-1}$ & $0.008 \pm 0.027$ & $-0.003 \pm 0.030$ & 0.007 \\
\hline$C_{5}^{1}$ & $-0.001 \pm 0.012$ & $-0.001 \pm 0.011$ & 0.951 \\
\hline$C_{5}{ }^{3}$ & $0.004 \pm 0.012$ & $0.005 \pm 0.014$ & 0.753 \\
\hline$C_{5}^{5}$ & $0.007 \pm 0.020$ & $0.008 \pm 0.023$ & 0.759 \\
\hline$C_{6}{ }^{-6}$ & $0.002 \pm 0.012$ & $0.002 \pm 0.015$ & 0.951 \\
\hline$C_{6}{ }^{-4}$ & $-0.006 \pm 0.010$ & $-0.007 \pm 0.008$ & 0.882 \\
\hline$C_{6}{ }^{-2}$ & $0.002 \pm 0.009$ & $-0.001 \pm 0.010$ & 0.053 \\
\hline$C_{6}{ }^{0}$ & $-0.027 \pm 0.016$ & $-0.030 \pm 0.018$ & 0.312 \\
\hline$C_{6}^{2}$ & $0.008 \pm 0.013$ & $0.008 \pm 0.017$ & 0.754 \\
\hline$C_{6}{ }^{4}$ & $-0.004 \pm 0.012$ & $-0.007 \pm 0.016$ & 0.186 \\
\hline$C_{6}^{6}$ & $-0.003 \pm 0.015$ & $-0.003 \pm 0.017$ & 0.904 \\
\hline
\end{tabular}

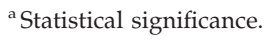

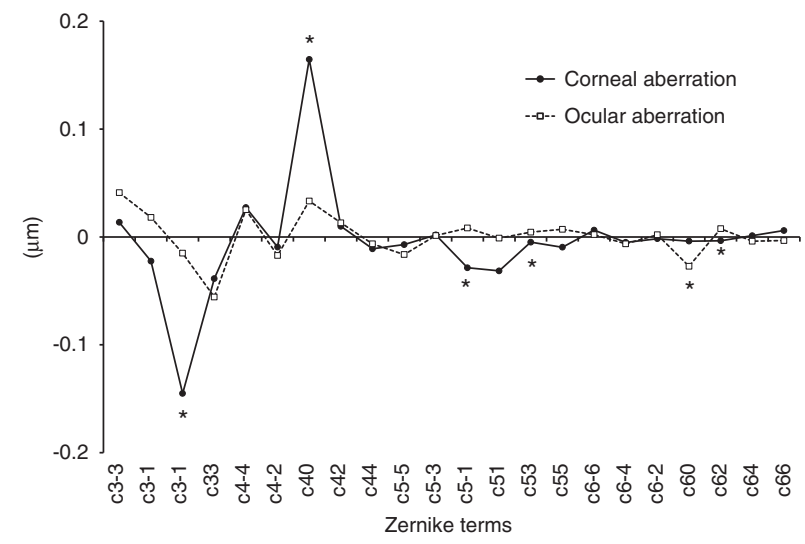

Figure 2 Comparison of individual Zernike components between corneal and ocular aberrations in a non-cycloplegic condition. In general, ocular aberration was smaller than or equivalent to corneal aberration. There were significant differences in $C_{3}{ }^{1}, C_{4}{ }^{0}, C_{5}{ }^{-1}, C_{5}{ }^{3}, C_{6}{ }^{0}$, and $C_{6}{ }^{2}$ between corneal and ocular aberrations $(P<0.002)$. Especially in horizontal coma $\left(C_{3}{ }^{1}\right)$ and spherical aberration $\left(C_{4}{ }^{0}\right)$, ocular aberration was much smaller than corneal aberration.

because of the balance between the sympathetic and parasympathetic innervation. ${ }^{22}$ Cyclopentolate relaxes the eye's normal functional state for distance-the tonic (steady-state) accommodation-by paralyzing the ciliary muscle. In our study, as expected, subjective myopic refractive error decreased by $0.50 \mathrm{D}$ after cycloplegia owing to the relaxation of tonic accommodation. Jankov et $a^{23}$ also confirmed that subjective cycloplegic sphere differed by $+0.24 \mathrm{D}$ (less myopic) from the subjective manifest sphere in myopic patients aged from 18 to 57 years.

In terms of second-order aberrations, there was also a difference between the conditions before and after the instillation of cyclopentolate. As might be expected, this difference was significant for the defocus term $C_{2}{ }^{0}$ $(P<0.001)$ but not in terms of vertical and horizontal astigmatism $C_{2}{ }^{2}(P=0.212)$ or oblique astigmatism $C_{2}{ }^{-2}$ $(P=0.625)$. As for ocular HOAs, spherical-like and total HOAs increased significantly after cycloplegia. Carkeet et $a l^{7}$ investigated the difference in ocular HOAs between cycloplegic and non-cycloplegic measurements in young adults aged from 18 to 27 years and showed that total HOAs in the cycloplegic condition were significantly higher than in the non-cycloplegic condition. Although subject age differed between the studies, our results supported the findings reported by Carkeet et al..$^{7}$ In contrast, Jankov et $a l^{23}$ reported that there was no significant change in total HOAs when cycloplegic and non-cycloplegic conditions were compared in adults. To our knowledge, there has been only one previous study investigating the changes in HOAs before and after cycloplegia with cyclopentolate in children. ${ }^{15}$ The researchers found no significant difference in HOAs before vs after cycloplegia and concluded that cycloplegia minimally affected HOAs in children aged 4-14 years. However, we found a significant increase in HOAs after cycloplegia with topical cyclopentolate in children. The discrepancy between studies may be attributable to the racial differences in ocular aberrations between Caucasians and Asians or the differences in time to the first measurements after the instillation of cyclopentolate $(30 \mathrm{~min}$ in the previous study vs $60 \mathrm{~min}$ in our study).

As for individual Zernike components, significant changes were found in $C_{2}{ }^{0}$ and $C_{4}{ }^{0}$. As described above, the decrease in defocus term $C_{2}{ }^{0}$ is reasonable. A positive shift in spherical aberration $\left(C_{4}{ }^{0}\right)$ after cycloplegia has been reported as well. ${ }^{23}$ In accordance with this, increasing levels of accommodation are associated with negative shifts in spherical aberration. ${ }^{10-13}$ These changes in spherical aberration with accommodation and cycloplegia are likely due to changes in the shape and motion of the crystalline lens. Cycloplegia is reported to induce significant decreases in the lens thickness and backward movement of the lens. ${ }^{24}$ Dubblman et al ${ }^{25,26}$ used Scheimpflug images to show that accommodation induces hypertrophy of the central portion of the lens. 
The authors reported that the radii of curvature of the anterior and posterior surfaces of the crystalline lens affected the change in spherical aberration, resulting in a late central wavefront. It is easy to imagine the opposite changes in lens shape and spherical aberration after cycloplegia. Our results are in good agreement with the findings reported by Jankov et $a l^{23}$ in terms of the positive direction of the change in spherical aberration after cycloplegia. This is the first study that confirmed the positive shift of spherical aberration after cycloplegia in myopic children.

The corneal HOAs did not change after cycloplegia. When corneal and ocular HOAs were compared, the corneal HOAs were significantly larger than the ocular HOAs, especially in spherical-like aberrations and total HOAs. Previous studies have demonstrated that the internal optics such as crystalline lens tend to compensate partly for corneal aberrations in young adults, producing an improved retinal image. ${ }^{10,27-29}$ However, in general, this compensation is partially or completely lost in older subjects owing to the increased HOAs of internal optics associated with aging. ${ }^{10}$ To our knowledge, the relationship between corneal and ocular HOAs has been well examined in segments of the population ranging in age from young adults to the elderly, ${ }^{10,27-29}$ but no information on the relationship in myopic children is available. Here, we report the novel finding that the whole eye had lower HOAs than the cornea in a pediatric population, which indicates that corneal HOAs are compensated for by internal optics also in myopic children. Although we recently found a similar result in hyperopic children, ${ }^{17}$ to the best of our knowledge, this is the first study that confirmed a compensation of corneal optics by the internal optics in myopic children.

It should be noted that all of the Zernike components exhibited large inter-individual variation. In addition, no significant correlation was observed among HOAs, refractive power, and age. The inter-individual variability in HOAs therefore outweighs any correlation with the nature of the patient's refractive error.

This study has certain limitations. Although we did not include emmetropes in the present study, some previous studies compared HOAs between myopic and emmetropic children. ${ }^{8,30} \mathrm{He}$ et al ${ }^{8}$ showed a significantly larger amount of HOAs in myopic as compared with emmetropic children and suggested that these higher levels of HOAs were a stimulus for the induction of myopia. In contrast, Carkeet et $a l^{30}$ reported that HOAs did not vary significantly between myopic and emmetropic children. Also in this point, no definitive conclusion is provided yet. Further research will be necessary to elucidate this issue. Another limitation is a possibility that the pupil dilation after administration of cyclopentolate and natural dilation without drugs result in slightly different positions for the pupil center with respect to the eye's optics. The shifting of pupil centers in this manner will cause a difference in aberration measurements between cycloplegic and non-cycloplegic conditions, and may affect the current results.

Although almost all previous studies have used the pupil center as a reference point, some researchers suggested that the use of an unvarying reference point (eg, the corneal limbus) may be better to avoid the above problem. ${ }^{7,31}$ This point should also be confirmed by another study.

In conclusion, we confirmed a significant increase in ocular HOAs after cycloplegia with cyclopentolate in myopic children. The ocular HOAs were significantly smaller than the corneal HOAs, indicating that internal optics compensated for the corneal HOAs. These findings imply that physiological tonic accommodation improves retinal image quality by reducing the wholeeye HOAs. Our study also showed that inter-individual variation in each Zernike component was large in myopic children.

\section{Summary}

\section{What was known before}

- There is little information in the literature about how cycloplegia with cyclopentolate affects the optical quality of the eye. Especially in children, ocular HOAs before and after cycloplegia have not been fully investigated.

What this study adds

- We confirmed a significant increase in ocular HOAs after cycloplegia with cyclopentolate in myopic children. The ocular HOAs were significantly smaller than the corneal HOAs, indicating that internal optics compensated for the corneal HOAs. Our study also showed that interindividual variation in each Zernike component was large in myopic children.

\section{Conflict of interest}

The authors declare no conflict of interest.

\section{References}

1 Campbell MC, Harrison EM, Simonet P. Psychophysical measurement of the blur on the retina due to optical aberrations of the eye. Vision Res 1990; 30(11): 1587-1602.

2 Atchison DA, Collins MJ, Wildsoet CF, Christensen J, Waterworth MD. Measurement of monochromatic ocular aberrations of human eyes as a function of accommodation by the Howland aberroscope technique. Vision Res 1995; 35(3): 313-323.

3 Liang J, Williams DR. Aberrations and retinal image quality of the normal human eye. J Opt Soc Am A Opt Image Sci Vis 1997; 14(11): 2873-2883. 
4 Amano S, Amano Y, Yamagami S, Miyai T, Miyata K, Samejima T et al. Age-related changes in corneal and ocular higher-order wavefront aberrations. Am J Ophthalmol 2004; 137(6): 988-992.

5 McLellan JS, Marcos S, Burns SA. Age-related changes in monochromatic wave aberrations of the human eye. Invest Ophthalmol Vis Sci 2001; 42(6): 1390-1395.

6 Brunette I, Bueno JM, Parent M, Hamam H, Simonet P. Monochromatic aberrations as a function of age, from childhood to advanced age. Invest Ophthalmol Vis Sci 2003; 44(12): 5438-5446.

7 Carkeet A, Velaedan S, Tan YK, Lee DY, Tan DT. Higher order ocular aberrations after cycloplegic and non-cycloplegic pupil dilation. J Refract Surg 2003; 19(3): 316-322.

8 He JC, Sun P, Held R, Thorn F, Sun X, Gwiazda JE. Wavefront aberrations in eyes of emmetropic and moderately myopic school children and young adults. Vision Res 2002; 42(8): 1063-1070.

9 Carkeet A, Leo SW, Khoo BK, Au Eong KG. Modulation transfer functions in children: pupil size dependence and meridional anisotropy. Invest Ophthalmol Vis Sci 2003; 44(7): 3248-3256.

10 He JC, Burns SA, Marcos S. Monochromatic aberrations in the accommodated human eye. Vision Res 2000; 40(1): 41-48.

11 Ninomiya S, Fujikado T, Kuroda T, Maeda N, Tano Y, Oshika T et al. Changes of ocular aberration with accommodation. Am J Ophthalmol 2002; 134(6): 924-926.

12 Cheng H, Barnett JK, Vilupuru AS, Marsack JD, Kasthurirangan S, Applegate RA et al. A population study on changes in wave aberrations with accommodation. $J$ Vis 2004; 4(4): 272-280.

13 Iida Y, Shimizu K, Ito M, Suzuki M. Influence of age on ocular wavefront aberration changes with accommodation. J Refract Surg 2008; 24(7): 696-701.

14 Ninomiya S, Fujikado T, Kuroda T, Maeda N, Tano Y, Hirohara $\mathrm{Y}$ et al. Wavefront analysis in eyes with accommodative spasm. Am J Ophthalmol 2003; 136(6): 1161-1163.

15 Kirwan C, O'Keefe M, Soeldner H. Higher-order aberrations in children. Am J Ophthalmol 2006; 141(1): 67-70.

16 Shah P, Jacks AS, Adams GG. Paediatric cycloplegia: a new approach. Eye (Lond) 1997; 11(6): 845-846.

17 Hiraoka T, Miyata K, Nakamura Y, Miyai T, Ogata M, Okamoto $\mathrm{F}$ et al. Influences of cycloplegia with topical atropine on ocular higher-order aberrations. Ophthalmology 2013; 120(1): 8-13.
18 Kuroda T, Fujikado T, Maeda N, Oshika T, Hirohara Y, Mihashi T. Wavefront analysis of higher-order aberrations in patients with cataract. J Cataract Refract Surg 2002; 28(3): 438-444.

19 Maeda N, Fujikado T, Kuroda T, Mihashi T, Hirohara Y, Nishida $\mathrm{K}$ et al. Wavefront aberrations measured with Hartmann-Shack sensor in patients with keratoconus. Ophthalmology 2002; 109(11): 1996-2003.

20 Fujikado T, Kuroda T, Ninomiya S, Maeda N, Tano Y, Oshika T et al. Age-related changes in ocular and corneal aberrations. Am J Ophthalmol 2004; 138(1): 143-146.

21 Zhao J, Mao J, Luo R, Li F, Pokharel GP, Ellwein LB. Accuracy of noncycloplegic autorefraction in school-age children in China. Optom Vis Sci 2004; 81(1): 49-55.

22 Gilmartin B. A review of the role of sympathetic innervation of the ciliary muscle in ocular accommodation. Ophthalmic Physiol Opt 1986; 6(1): 23-37.

23 Jankov 2nd MR, Iseli HP, Bueeler M, Schor P, Seiler T, Mrochen M. The effect of phenylephrine and cyclopentolate on objective wavefront measurements. J Refract Surg 2006; 22(5): 472-481.

24 Gao L, Zhuo X, Kwok AK, Yu N, Ma L, Wang J. The change in ocular refractive components after cycloplegia in children. Jpn J Ophthalmol 2002; 46(3): 293-298.

25 Dubbelman M, Van der Heijde GL, Weeber HA, Vrensen GF. Changes in the internal structure of the human crystalline lens with age and accommodation. Vision Res 2003; 43(22): 2363-2375.

26 Dubbelman M, Van der Heijde GL, Weeber HA. Change in shape of the aging human crystalline lens with accommodation. Vision Res 2005; 45(1): 117-132.

27 El-Hage SG, Berny F. Contribution of the crystalline lens to the spherical aberration of the eye. J Opt Soc Am 1973; 63(2): 205-211.

28 Artal P, Guirao A. Contributions of the cornea and the lens to the aberrations of the human eye. Opt Lett 1998; 23(21): 1713-1715.

29 Artal P, Guirao A, Berrio E, Williams DR. Compensation of corneal aberrations by the internal optics in the human eye. J Vis 2001; 1(1): 1-8.

30 Carkeet A, Luo HD, Tong L, Saw SM, Tan DT. Refractive error and monochromatic aberrations in Singaporean children. Vision Res 2002; 42(14): 1809-1824.

31 Yang Y, Thompson K, Burns SA. Pupil location under mesopic, photopic, and pharmacologically dilated conditions. Invest Ophthalmol Vis Sci 2002; 43(7): 2508-2512. 